Supplement of Solid Earth, 10, 741-763, 2019

https://doi.org/10.5194/se-10-741-2019-supplement

(C) Author(s) 2019. This work is distributed under

the Creative Commons Attribution 4.0 License.

(c) (1)

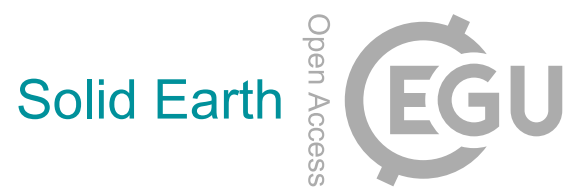

Supplement of

\title{
The Bortoluzzi Mud Volcano (Ionian Sea, Italy) and its potential for tracking the seismic cycle of active faults
}

Marco Cuffaro et al.

Correspondence to: Andrea Billi (andrea.billi@cnr.it)

The copyright of individual parts of the supplement might differ from the CC BY 4.0 License. 


\section{Introduction}

Supporting material contains two tables and three figures as supplement to the main text. 


\begin{tabular}{|c|c|c|c|c|c|c|}
\hline $\begin{array}{c}\text { Date } \\
\text { (yyyymmdd) }\end{array}$ & $\begin{array}{c}\text { UTC Time } \\
\text { (hh:mm:ss.cc) }\end{array}$ & $\begin{array}{c}\text { Latitude } \\
\left({ }^{\circ} \mathbf{N}\right)\end{array}$ & $\begin{array}{c}\text { Longitude } \\
\left({ }^{\circ} \mathbf{E}\right)\end{array}$ & $\begin{array}{c}\text { Depth } \\
(\mathbf{k m})\end{array}$ & Md & Ml \\
\hline 19851121 & 23:56:32.16 & 37.8955 & 16.0202 & 0.03 & 2.6 & \\
\hline 19851229 & 12:18:00.51 & 37.6732 & 16.4792 & 11.26 & 3.0 & \\
\hline 19860201 & $13: 44: 55.95$ & 37.6853 & 16.0015 & 27.58 & 2.5 & \\
\hline 19870419 & $12: 30: 31.97$ & 37.7972 & 16.1732 & 3.07 & 3.4 & \\
\hline 19870419 & 19:44:15.95 & 37.8780 & 16.1058 & 0.2 & 2.9 & \\
\hline 19880603 & $12: 41: 43.10$ & 37.6802 & 16.5248 & 31.16 & 2.5 & 2.0 \\
\hline 19880801 & 19:28:15.68 & 37.8808 & 16.3282 & 29.51 & 3.0 & 2.2 \\
\hline 19881023 & $13: 41: 54.96$ & 37.9208 & 16.0888 & 0.15 & 2.4 & \\
\hline 19890113 & $11: 56: 31.38$ & 37.7672 & 16.2522 & 39.92 & 2.6 & 2.2 \\
\hline 19890613 & 21:13:55.06 & 38.0547 & 16.3642 & 11.25 & 2.8 & 1.9 \\
\hline 19890717 & $13: 57: 25.70$ & 37.6060 & 16.4513 & 28.34 & 2.6 & 2.2 \\
\hline 19891029 & $12: 44: 49.93$ & 37.8642 & 16.3093 & 31.78 & 2.4 & 1.8 \\
\hline 19900306 & $20: 51: 22.54$ & 37.7282 & 16.2113 & 25.89 & 2.4 & 1.8 \\
\hline 19900325 & $22: 30: 42.18$ & 37.6903 & 16.2258 & 32.69 & 2.3 & 1.9 \\
\hline 19901006 & 05:52:38.98 & 37.9513 & 16.0980 & 29.55 & 2.3 & \\
\hline 19910116 & $20: 18: 10.45$ & 37.9718 & 16.0163 & 7.37 & 2.9 & 2.8 \\
\hline 19910716 & $00: 41: 36.05$ & 38.0713 & 16.2038 & 10.92 & 2.4 & \\
\hline 19911011 & $00: 14: 41.59$ & 37.6447 & 16.3388 & 34.07 & 3.2 & 2.8 \\
\hline 19951007 & $05: 41: 58.79$ & 38.0785 & 16.0225 & 10.61 & 3.2 & 2.8 \\
\hline 20021201 & $02: 17: 43.59$ & 37.8757 & 16.1337 & 4.83 & 2.4 & \\
\hline 20021202 & $02: 22: 04.57$ & 37.8272 & 16.2090 & 4.47 & 2.7 & \\
\hline 20030103 & 11:44:04.03 & 37.6185 & 16.1377 & 18.23 & 2.5 & \\
\hline 20030312 & $05: 49: 22.86$ & 38.0772 & 16.0022 & 5.43 & 3.0 & 2.3 \\
\hline 20030612 & $18: 51: 27.04$ & 37.6082 & 16.4098 & 28.63 & 3.2 & 3.5 \\
\hline 20030709 & $22: 19: 17.19$ & 37.9750 & 16.2527 & 14.31 & 2.6 & 1.7 \\
\hline 20031210 & 06:38:05.47 & 37.6133 & 16.3245 & 33.01 & 2.6 & 2.6 \\
\hline 20040704 & $06: 49: 17.93$ & 38.0018 & 16.0557 & 5.86 & 2.4 & 2.0 \\
\hline 20040704 & $08: 46: 25.71$ & 37.9848 & 16.1170 & 6.92 & 2.3 & 1.9 \\
\hline 20040707 & 19:16:57.13 & 37.9955 & 16.1187 & 3.01 & 2.3 & 2.1 \\
\hline 20040715 & $21: 17: 20.34$ & 38.0177 & 16.0382 & 3.35 & 2.7 & 2.5 \\
\hline 20040717 & $16: 43: 35.49$ & 37.9212 & 16.4295 & 4.29 & 2.8 & 2.6 \\
\hline 20040816 & $03: 13: 29.43$ & 37.7943 & 16.2753 & 33.96 & 2.5 & 2.0 \\
\hline 20041117 & $04: 24: 32.43$ & 38.0408 & 16.0392 & 6.44 & 2.5 & 2.4 \\
\hline 20050323 & $21: 59: 25.45$ & 37.6413 & 16.2912 & 30.28 & 2.5 & 2.0 \\
\hline 20050503 & $18: 41: 12.50$ & 37.9628 & 16.0937 & 10.01 & 1.7 & \\
\hline 20050503 & 19:34:49.90 & 38.0080 & 16.1682 & 9.58 & 1.6 & \\
\hline 20050513 & 14:31:19.96 & 37.7543 & 16.1645 & 32.59 & 2.1 & \\
\hline 20050617 & 20:53:21.97 & 38.0817 & 16.1515 & 13.11 & 2.6 & \\
\hline 20050910 & $00: 48: 59.05$ & 38.0958 & 16.2702 & 12.1 & 1.7 & \\
\hline 20051002 & $01: 12: 32.81$ & 37.7655 & 16.0492 & 34.38 & 2.2 & \\
\hline
\end{tabular}




\begin{tabular}{|c|c|c|c|c|c|c|}
\hline 20060421 & $18: 12: 56.85$ & 37.6153 & 16.2015 & 28.15 & 1.9 & \\
\hline 20060530 & $11: 30: 40.54$ & 37.6212 & 16.5143 & 30.02 & 4.2 & \\
\hline 20060530 & $11: 38: 34.44$ & 37.6163 & 16.4632 & 32.76 & 3.5 & \\
\hline 20060530 & $13: 02: 51.33$ & 37.6008 & 16.4608 & 31.84 & 2.4 & \\
\hline 20060530 & $22: 16: 54.00$ & 37.6145 & 16.4350 & 32.84 & 2.7 & \\
\hline 20060531 & $13: 56: 57.70$ & 37.6155 & 16.4372 & 27.1 & 2.9 & \\
\hline 20060619 & $00: 14: 23.17$ & 37.6525 & 16.3210 & 31.01 & 2.0 & \\
\hline 20060720 & 03:48:05.59 & 37.6342 & 16.1395 & 27.91 & 2.1 & \\
\hline 20060730 & $09: 53: 36.02$ & 38.0093 & 16.2888 & 10.17 & 2.8 & \\
\hline 20060730 & $10: 04: 58.24$ & 38.0040 & 16.2828 & 11.95 & 1.6 & \\
\hline 20060801 & $22: 09: 47.40$ & 37.7652 & 16.0215 & 25.69 & 1.9 & \\
\hline 20060830 & $07: 53: 55.36$ & 37.6597 & 16.2517 & 27.97 & 2.8 & \\
\hline 20061003 & $04: 58: 28.51$ & 37.7792 & 16.2588 & 11.65 & 2.6 & \\
\hline 20061229 & $12: 24: 32.87$ & 37.6073 & 16.0407 & 34.82 & 2.2 & \\
\hline 20070310 & $19: 52: 16.52$ & 38.0255 & 16.1078 & 35.94 & 2.3 & \\
\hline 20070421 & $06: 15: 43.47$ & 37.6903 & 16.3018 & 30.21 & 3.0 & \\
\hline 20070515 & $02: 44: 25.39$ & 37.7540 & 16.1197 & 22.04 & 2.2 & \\
\hline 20070602 & $04: 33: 34.97$ & 37.7773 & 16.1377 & 26.16 & 2.0 & \\
\hline 20070613 & 10:08:01.78 & 38.0175 & 16.4470 & 31.28 & 1.6 & \\
\hline 20070619 & $12: 41: 06.37$ & 37.6733 & 16.4775 & 26.96 & 1.9 & \\
\hline 20070623 & 19:19:22.61 & 37.6175 & 16.2180 & 18.44 & 1.9 & \\
\hline 20070702 & $23: 05: 24.50$ & 38.0005 & 16.5470 & 38.22 & 1.6 & \\
\hline 20070803 & $04: 14: 22.74$ & 37.9570 & 16.3065 & 19.3 & 2.1 & \\
\hline 20070803 & $07: 17: 17.05$ & 37.9750 & 16.3172 & 16.32 & 2.0 & \\
\hline 20070803 & $12: 42: 02.04$ & 37.9415 & 16.3312 & 7.19 & 2.4 & \\
\hline 20070803 & $16: 54: 20.35$ & 38.0090 & 16.2393 & 27.95 & 1.7 & \\
\hline 20070816 & $22: 24: 49.65$ & 37.8882 & 16.4192 & 25.93 & 1.6 & \\
\hline 20070913 & $14: 45: 41.50$ & 37.9733 & 16.3315 & 10.85 & 1.7 & \\
\hline 20070913 & $17: 19: 10.75$ & 37.8937 & 16.3368 & 10.01 & 2.9 & \\
\hline 20080110 & 09:02:57.10 & 37.6312 & 16.0713 & 27.99 & 2.2 & \\
\hline 20080116 & $19: 43: 46.68$ & 37.7818 & 16.2277 & 34.33 & 2.7 & \\
\hline 20080120 & 01:50:50.97 & 38.0550 & 16.0753 & 14.64 & 1.5 & \\
\hline 20080124 & $12: 05: 31.75$ & 38.0537 & 16.0717 & 14.31 & 1.6 & \\
\hline 20080201 & $19: 10: 23.25$ & 37.9010 & 16.0245 & 5.81 & 2.1 & \\
\hline 20080306 & $11: 05: 57.50$ & 38.0822 & 16.2000 & 14.81 & 1.6 & \\
\hline 20080306 & $15: 53: 41.33$ & 38.0747 & 16.2203 & 15.79 & 2.1 & \\
\hline 20080308 & $04: 51: 52.43$ & 38.0873 & 16.2045 & 12 & 1.8 & \\
\hline 20080509 & $04: 34: 50.80$ & 37.6428 & 16.1038 & 29.12 & 1.7 & \\
\hline 20080726 & 09:10:30.31 & 37.8418 & 16.1820 & 39.9 & 2.1 & \\
\hline 20081020 & 09:10:31.02 & 37.8638 & 16.0417 & 5.49 & 1.7 & 2.2 \\
\hline 20081102 & $06: 46: 43.72$ & 37.6338 & 16.4657 & 33.11 & 3.4 & 1.7 \\
\hline 20090128 & $00: 21: 49.93$ & 38.0720 & 16.1932 & 7.07 & 3.0 & 2.3 \\
\hline 20090310 & $20: 33: 05.37$ & 38.0628 & 16.2238 & 10.25 & 2.0 & 2.3 \\
\hline 20090311 & $04: 51: 56.64$ & 37.8397 & 16.4040 & 29.59 & 2.0 & 3.1 \\
\hline 20090425 & $10: 15: 35.96$ & 37.6247 & 16.3542 & 33.06 & 3.1 & \\
\hline 20090526 & 11:04:12.44 & 37.9940 & 16.2785 & 13.73 & 1.8 & \\
\hline
\end{tabular}




\begin{tabular}{|c|c|c|c|c|c|}
\hline 20090528 & $02: 55: 53.04$ & 37.9652 & 16.1055 & 6.64 & 1.2 \\
\hline 20090604 & $18: 47: 59.08$ & 37.6580 & 16.2803 & 31.85 & 1.7 \\
\hline 20090628 & $01: 31: 58.47$ & 37.6327 & 16.0060 & 17.54 & 1.7 \\
\hline 20090821 & 14:07:03.33 & 37.8625 & 16.0960 & 31.1 & 1.5 \\
\hline 20090930 & $19: 55: 56.43$ & 37.9765 & 16.0303 & 7.01 & 1.0 \\
\hline 20091013 & $22: 25: 37.36$ & 37.7257 & 16.1983 & 28.06 & 2.1 \\
\hline 20091109 & $18: 45: 28.58$ & 38.0713 & 16.4277 & 10.16 & 1.9 \\
\hline 20091109 & $19: 31: 53.15$ & 38.0772 & 16.4210 & 15.41 & 1.5 \\
\hline 20091110 & $04: 40: 25.49$ & 38.0803 & 16.4190 & 9.96 & 2.7 \\
\hline 20091110 & $07: 14: 02.93$ & 38.0995 & 16.4017 & 10.42 & 2.2 \\
\hline 20091111 & $22: 51: 11.81$ & 38.0855 & 16.4263 & 9.27 & 2.5 \\
\hline 20091113 & $08: 28: 21.83$ & 38.0543 & 16.4447 & 10.85 & 1.5 \\
\hline 20091116 & 14:08:10.71 & 38.0723 & 16.4315 & 12.5 & 1.6 \\
\hline 20091118 & $00: 56: 00.18$ & 38.0720 & 16.4335 & 15.6 & 1.3 \\
\hline 20091118 & $06: 56: 03.25$ & 38.0767 & 16.4333 & 15.7 & 1.5 \\
\hline 20091118 & 10:58:15.09 & 38.0885 & 16.4028 & 10.01 & 2.1 \\
\hline 20091118 & $15: 48: 31.29$ & 38.1027 & 16.3968 & 4.59 & 2.6 \\
\hline 20091120 & 10:14:00.21 & 38.0717 & 16.4458 & 10.15 & 1.3 \\
\hline 20091121 & $10: 27: 49.12$ & 38.0843 & 16.4135 & 10.25 & 2.2 \\
\hline 20091125 & 06:20:07.09 & 38.0593 & 16.4503 & 13.92 & 3.1 \\
\hline 20091211 & $06: 34: 17.61$ & 38.0813 & 16.4310 & 12.13 & 2.0 \\
\hline 20091211 & $08: 28: 21.74$ & 38.0797 & 16.4287 & 15.88 & 1.9 \\
\hline 20091211 & 09:02:33.58 & 38.0735 & 16.4312 & 12.44 & 2.1 \\
\hline 20091220 & $05: 53: 38.96$ & 38.0760 & 16.4228 & 15.63 & 2.1 \\
\hline 20091223 & $02: 11: 59.85$ & 38.0783 & 16.4107 & 9.7 & 2.7 \\
\hline 20091225 & $16: 37: 55.26$ & 38.0993 & 16.4158 & 4.83 & 1.5 \\
\hline 20091229 & 03:31:01.45 & 37.6932 & 16.1437 & 34.97 & 2.3 \\
\hline 20100403 & $21: 20: 50.93$ & 37.7162 & 16.0773 & 18.42 & 1.6 \\
\hline 20110527 & $08: 29: 23.10$ & 37.9240 & 16.0108 & 33.47 & 2.0 \\
\hline 20110601 & $07: 56: 04.00$ & 37.6580 & 16.1067 & 29.09 & 2.3 \\
\hline 20110803 & $02: 44: 49.90$ & 37.7717 & 16.0723 & 21.64 & 1.6 \\
\hline 20110819 & $10: 10: 13.36$ & 37.9337 & 16.0155 & 4.14 & 2.0 \\
\hline 20120422 & $08: 30: 52.98$ & 37.7508 & 16.2737 & 37.92 & 1.8 \\
\hline 20120630 & $21: 45: 28.94$ & 37.8802 & 16.2077 & 15.04 & 2.0 \\
\hline 20120702 & $05: 02: 32.47$ & 38.0613 & 16.0263 & 4.6 & 2.0 \\
\hline 20120714 & $09: 42: 43.70$ & 38.0565 & 16.1310 & 2.84 & 1.6 \\
\hline 20120717 & $11: 11: 15.44$ & 37.9418 & 16.2212 & 15.69 & 2.4 \\
\hline 20120717 & $11: 17: 36.39$ & 38.0307 & 16.2403 & 12.28 & 2.4 \\
\hline 20120717 & $11: 19: 55.25$ & 38.0907 & 16.2852 & 4.98 & 2.5 \\
\hline 20120717 & $11: 22: 39.11$ & 37.9877 & 16.2640 & 23.42 & 2.5 \\
\hline 20120718 & $01: 31: 50.62$ & 38.0187 & 16.2615 & 17.4 & 2.4 \\
\hline 20120718 & 11:17:11.04 & 37.8852 & 16.1667 & 15.6 & 2.6 \\
\hline 20120726 & $09: 53: 34.12$ & 37.6583 & 16.0908 & 24.03 & 1.9 \\
\hline 20120726 & $14: 20: 03.13$ & 37.9168 & 16.3243 & 3.06 & 3.0 \\
\hline 20120731 & $07: 27: 30.42$ & 38.0270 & 16.1970 & 22.62 & 2.1 \\
\hline 20120802 & 00:02:07.16 & 38.0568 & 16.2698 & 4.43 & 2.2 \\
\hline
\end{tabular}




\begin{tabular}{|c|c|c|c|c|c|}
\hline 20119902 & 18:00:20.34 & 38.0373 & 16.3210 & 3.98 & 2.8 \\
\hline 20121002 & $00: 47: 43.19$ & 37.9760 & 16.0265 & 7.85 & 1.9 \\
\hline 20121003 & $04: 24: 34.04$ & 37.9090 & 16.0355 & 8.26 & 1.8 \\
\hline 20121118 & $02: 05: 29.55$ & 37.9948 & 16.2310 & 21.54 & 1.9 \\
\hline 20130120 & $01: 30: 13.39$ & 37.6863 & 16.2873 & 30.95 & 2.1 \\
\hline 20130201 & $20: 36: 20.60$ & 37.7533 & 16.1885 & 31.26 & 2.1 \\
\hline 20130312 & $13: 49: 42.94$ & 37.7528 & 16.2953 & 28.01 & 2.4 \\
\hline 20130324 & $15: 47: 21.81$ & 37.6783 & 16.4573 & 28.47 & 4.4 \\
\hline 20130324 & $20: 37: 50.10$ & 37.7062 & 16.3175 & 30.54 & 3.1 \\
\hline 20130516 & $15: 40: 48.90$ & 37.8337 & 16.2905 & 29.06 & 2.9 \\
\hline 20130721 & $23: 41: 15.28$ & 37.7438 & 16.4678 & 31.62 & 2.0 \\
\hline 20130724 & 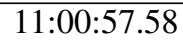 & 37.8597 & 16.5353 & 33.7 & 2.1 \\
\hline 20130731 & $02: 30: 59.02$ & 37.7402 & 16.2977 & 28 & 2.1 \\
\hline 20130921 & $15: 17: 47.72$ & 37.7873 & 16.3537 & 28.69 & 2.5 \\
\hline 20131216 & $23: 08: 43.28$ & 38.0543 & 16.2352 & 14.59 & 2.8 \\
\hline 20140102 & $14: 36: 00.51$ & 37.6815 & 16.1115 & 28.06 & 2.1 \\
\hline 20140118 & $06: 17: 41.12$ & 37.8860 & 16.1418 & 33.62 & 3.1 \\
\hline 20140121 & $03: 33: 36.12$ & 37.9937 & 16.1230 & 6.49 & 2.3 \\
\hline 20140703 & $22: 55: 38.30$ & 37.7743 & 16.1965 & 13.65 & 2.0 \\
\hline 20140709 & $22: 09: 40.40$ & 37.7048 & 16.1087 & 27.64 & 2.1 \\
\hline 20140721 & 01:05:18.93 & 37.8912 & 16.4278 & 33.16 & 2.4 \\
\hline 20140721 & $18: 22: 17.95$ & 37.6978 & 16.3008 & 29.73 & 2.7 \\
\hline 20140728 & $21: 57: 54.99$ & 37.8240 & 16.1790 & 29.79 & 2.3 \\
\hline 20141109 & $21: 08: 24.14$ & 37.8610 & 16.2615 & 33.1 & 2.1 \\
\hline 20141112 & $06: 27: 41.83$ & 37.6362 & 16.1432 & 25.23 & 2.1 \\
\hline 20150214 & $05: 31: 41.43$ & 37.7132 & 16.1563 & 29.98 & 2.2 \\
\hline 20150328 & $08: 21: 54.39$ & 37.6077 & 16.1653 & 27.99 & 3.1 \\
\hline 20150419 & $14: 05: 45.94$ & 37.6068 & 16.0017 & 28.01 & 2.4 \\
\hline 20150604 & $08: 49: 48.55$ & 37.7353 & 16.1658 & 31.22 & 2.3 \\
\hline 20150708 & $13: 43: 21.89$ & 37.7795 & 16.2287 & 32.72 & 2.7 \\
\hline 20150721 & 10:00:46.67 & 37.6920 & 16.3807 & 28.85 & 2.0 \\
\hline 20150930 & $03: 26: 47.68$ & 37.7982 & 16.3145 & 23.01 & 2.0 \\
\hline 20151004 & 17:08:08.98 & 37.6908 & 16.4070 & 29.2 & 2.2 \\
\hline 20151121 & $17: 53: 12.98$ & 37.6332 & 16.1707 & 28.08 & 2.1 \\
\hline 20151122 & $10: 02: 59.11$ & 37.6590 & 16.4327 & 28.03 & 2.5 \\
\hline 20151124 & $22: 29: 23.54$ & 37.6287 & 16.3213 & 28.19 & 2.2 \\
\hline 20160103 & $23: 55: 20.58$ & 37.7265 & 16.1963 & 37.06 & 2.4 \\
\hline 20160111 & 01:07:40.45 & 37.8003 & 16.0587 & 15.26 & 2.3 \\
\hline 20160112 & $05: 24: 28.71$ & 37.7713 & 16.4463 & 28.04 & 2.5 \\
\hline 20160228 & $01: 11: 45.33$ & 37.6797 & 16.0725 & 35.49 & 2.1 \\
\hline 20160509 & $15: 50: 16.29$ & 37.6307 & 16.3672 & 33.72 & 2.5 \\
\hline 20160514 & $10: 47: 18.20$ & 37.6343 & 16.4138 & 30.91 & 2.6 \\
\hline 20160821 & $21: 27: 42.58$ & 37.8805 & 16.0333 & 33.3 & 2.4 \\
\hline 20160927 & $07: 16: 29.18$ & 37.7863 & 16.2637 & 22.9 & 2.5 \\
\hline 20161101 & $04: 32: 27.48$ & 37.6427 & 16.4178 & 27.98 & 2.4 \\
\hline 20170211 & 00:04:30.95 & 37.6813 & 16.2428 & 27.7 & 2.2 \\
\hline
\end{tabular}


Table S1: Data for earthquakes in the BMV area shown as epicenters in Figs. 1 and 2(a) and as hypocenters in Fig. 2b. Data are from the public database of INGV Centro Nazionale Terremoti (http://cnt.rm.ingv.it/), relocated by one of us (T.S.). See text for further details. 


\begin{tabular}{|c|c|c|c|c|c|c|c|c|c|}
\hline $\mathbf{N}^{\circ}$ & $\begin{array}{c}\text { Date } \\
(\text { yyyymmdd) }\end{array}$ & $\begin{array}{c}\text { UTC Time } \\
(\text { hh:mm:ss.cc) }\end{array}$ & $\begin{array}{c}\text { Latitude } \\
\left({ }^{\circ} \mathbf{N}\right)\end{array}$ & $\begin{array}{c}\text { Longitude } \\
\left({ }^{\circ} \mathbf{E}\right)\end{array}$ & $\begin{array}{c}\text { Depth } \\
(\mathbf{k m})\end{array}$ & $\begin{array}{c}\text { Strike } \\
\left({ }^{\circ}\right)\end{array}$ & $\begin{array}{c}\text { Dip } \\
\left({ }^{\circ}\right)\end{array}$ & $\begin{array}{c}\text { Rake } \\
\left({ }^{\circ}\right)\end{array}$ & Mw \\
\hline 6 & 20060730 & $09: 53: 35.90$ & 37.99 & 16.31 & 6.0 & 292 & 64 & -7 & 2.70 \\
\hline 15 & 20090316 & $00: 28: 05.90$ & 37.67 & 15.96 & 28.0 & 34 & 60 & -24 & 3.00 \\
\hline 20 & 20091125 & $06: 20: 07.30$ & 38.05 & 16.45 & 16.0 & 341 & 62 & -43 & 3.20 \\
\hline 29 & 20120726 & $14: 20: 03.00$ & 37.90 & 16.34 & 16.0 & 134 & 83 & -19 & 3.10 \\
\hline 2b & 20130324 & $15: 47: 22.00$ & 37.76 & 16.50 & 30.0 & 257 & 87 & 178 & 4.56 \\
\hline
\end{tabular}

Table S2: Data for the earthquake focal mechanisms shown in Figs. 1 and 2. Data are from the European-Mediterranean Regional Centroid-Moment Tensors (RCMT) catalog (http://rcmt2.bo.ingv.it/) (red beach ball in Figs. 1 and 2) and from previous papers (blue beach balls in Figs. 1 and 2) by Orecchio et al. (2014) and Polonia et al. (2016). $\mathrm{N}^{\circ}$ refers to the numbers shown in Figs. 1 and 2 close to each focal mechanism. 


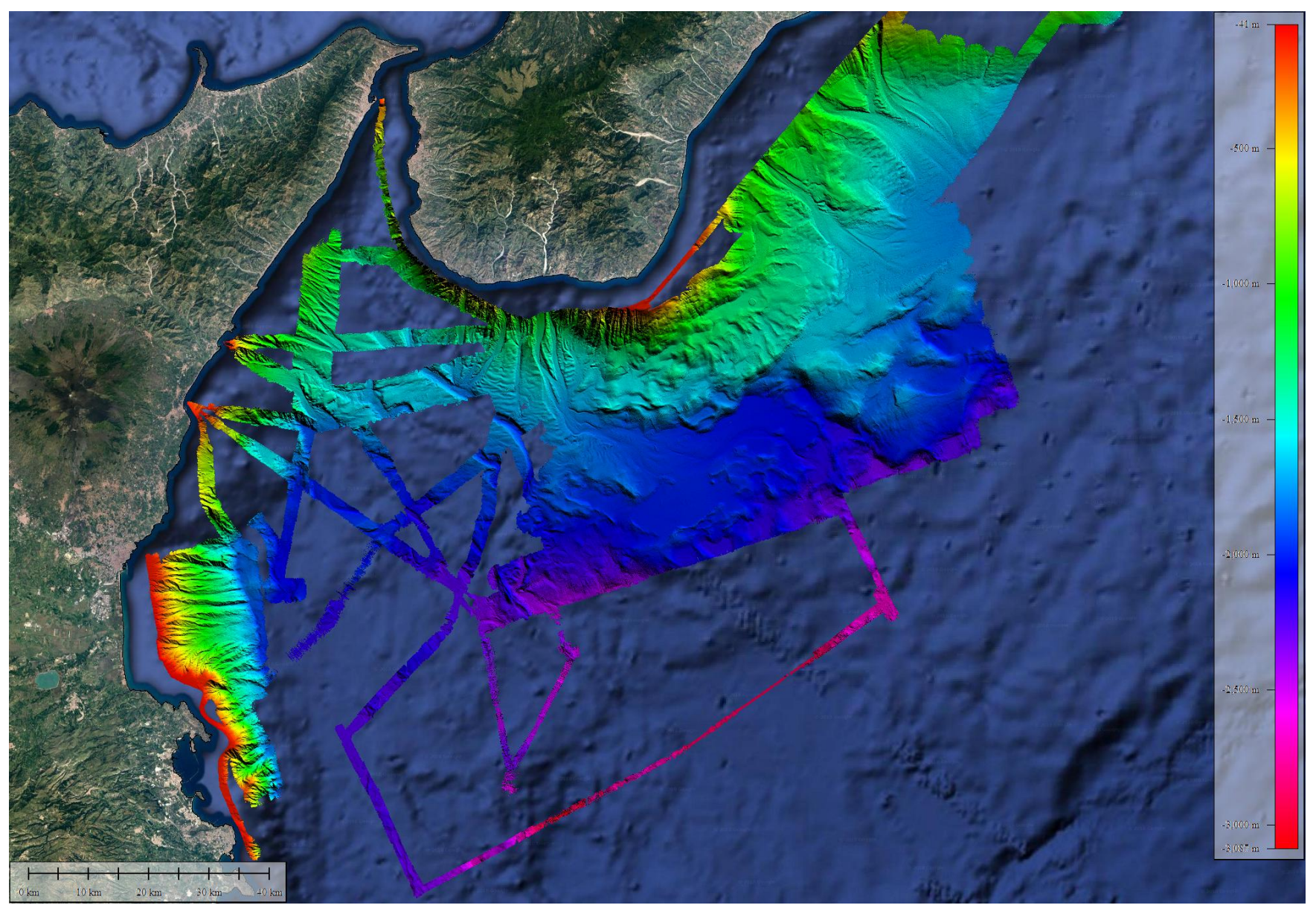

Figure S1. High-resolution multibeam bathymetry of the Ionian Sea acquired during the Seismofaults 2017 cruise. Note that, with this work, we will freely release the raw numerical data for $4970 \mathrm{~km}^{2}$ of newly-acquired high-resolution bathymetric data (graphically shown in this figure) for the Ionian Sea including the BMV. The raw data are externally hosted and indexed with doi: XXXX. 

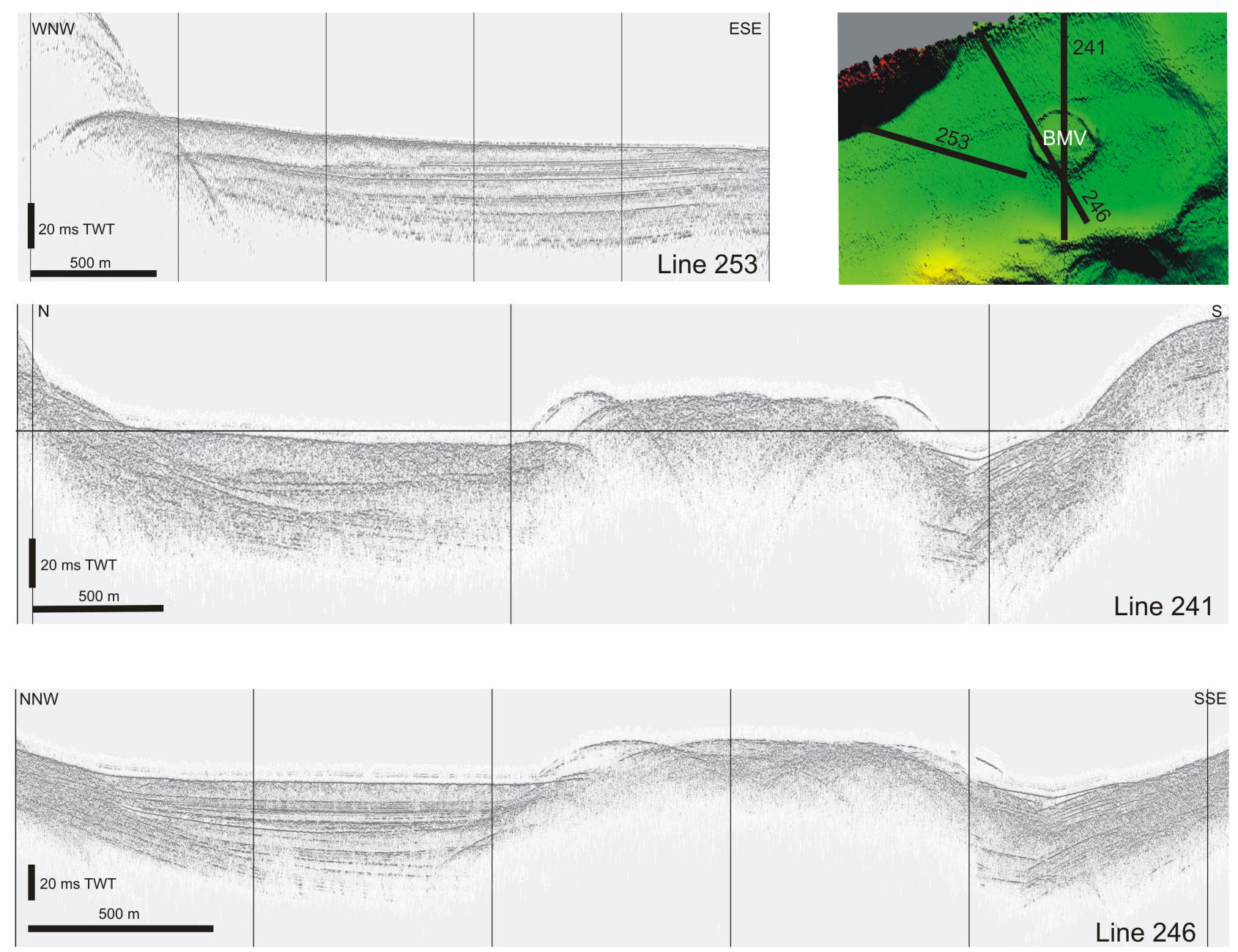

Figure S2. Non-interpreted high-resolution version of Fig. 7. 


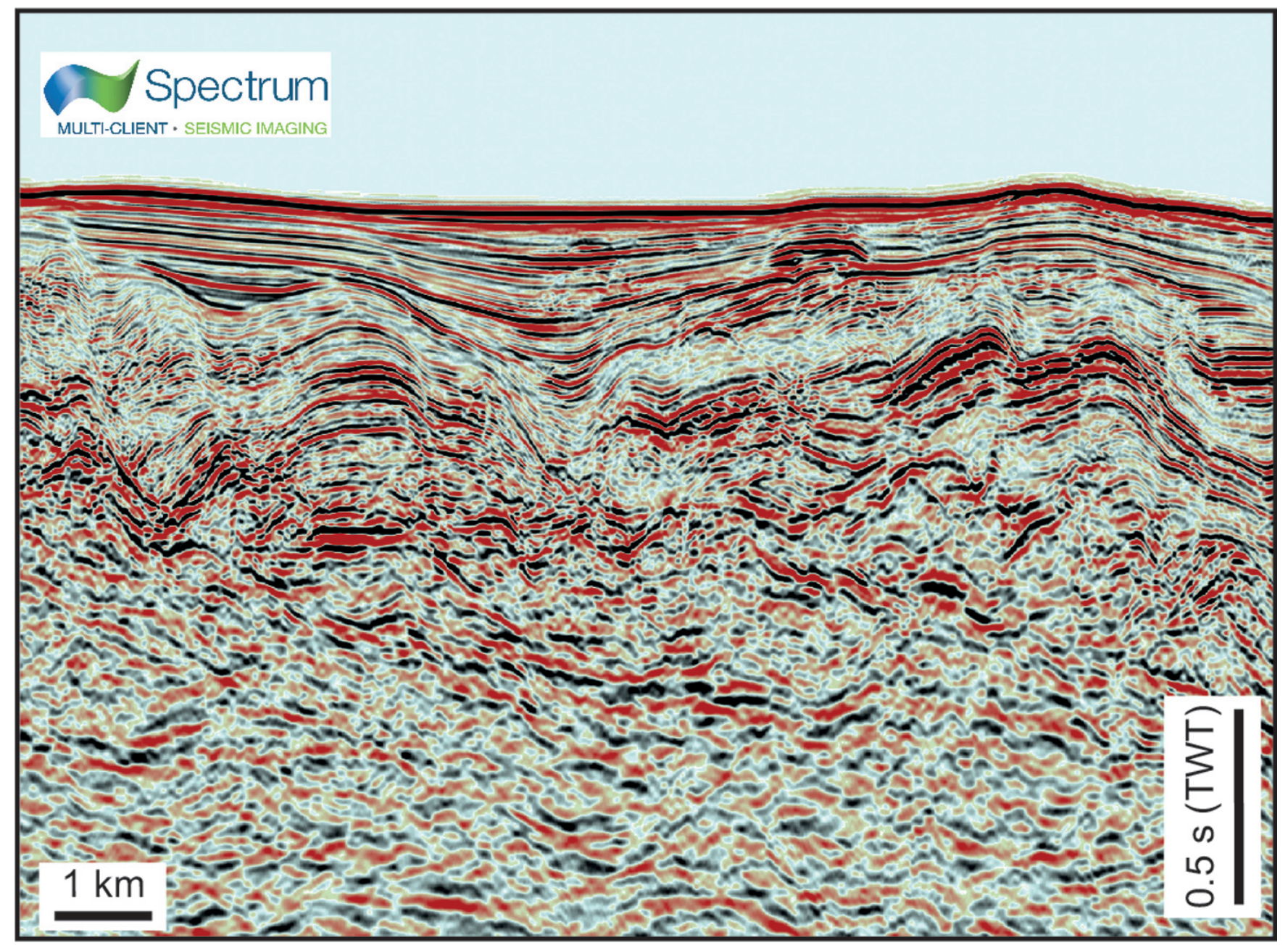

Figure S3. Non-interpreted high-resolution seismic reflection image shown in Fig. 10(e). 